\title{
Optical spectra of selected Chamaeleon I young stellar objects ${ }^{\star}$
}

\author{
C. Saffe ${ }^{1, \star \star}$, M. Gómez ${ }^{1, \star \star \star}$, S. Randich ${ }^{2}$, D. Mardones ${ }^{3}$, P. Caselli ${ }^{2}$, P. Persi ${ }^{4}$, and G. Racca ${ }^{1}$ \\ 1 Observatorio Astronómico de Córdoba, Laprida 854, 5000 Córdoba, Argentina \\ e-mail: saffe@oac.uncor.edu; mercedes@oac.uncor.edu; german@oac.uncor.edu \\ 2 INAF-Osservatorio Astrofisico di Arcetri, L.go E. Fermi, 550125 Firenze, Italia \\ e-mail: randich@arcetri.astro.it; caselli@arcetri.astro.it \\ 3 Departamento de Astronomía, Universidad de Chile, Casilla 36-D, Santiago, Chile \\ e-mail: mardones@das.uchile.cl \\ ${ }^{4}$ Istituto Astrofisica Spaziale e Fisica Cosmica, CNR, Via del Fosso del Cavaliere 00133, Roma, Italia \\ e-mail: persi@rm.iasf.cnr.it
}

Received 25 April 2003 / Accepted 22 July 2003

\begin{abstract}
We present optical spectra of eight candidate brown dwarfs and a previously known T Tauri star (Sz 33) of the Chamaeleon I dark cloud. We derived spectral types based on the strength of the TiO or VO absorption bands present in the spectra of these objects as well as on the PC3 index of Martín et al. (1999). Photometric data from the literature are used to estimate the bolometric luminosities for these sources. We apply D'Antona \& Mazzitelli (1997) pre-main sequence evolutionary tracks and isochrones to derive masses and ages. Based on the presence of $\mathrm{H} \alpha$ in emission, we confirm that most of the candidates are young objects. Our sample however includes two sources for which we can only provide upper limits for the emission in $\mathrm{H} \alpha$; whereas these two objects are most likely foreground/background stars, higher resolution spectra are required to confirm their true nature. Among the likely cloud members, we detect one new sub-stellar object and three transition stellar/sub-stellar sources.
\end{abstract}

Key words. stars: pre-main sequence - stars: Hertzsprung-Russell (HR) diagram - stars: low-mass, brown dwarfs infrared: stars

\section{Introduction}

The field of star and planet formation has attracted much observational and theoretical interest, particularly during the last twenty years. Impressive technological advances have reached high spatial resolution and sensitivity limits and allowed us to observe true stellar embryos and their circumstellar environments (disks), where planets are supposed to form. Brown dwarfs (BDs) represent an intermediate class of objects between stars and planets. They have masses below the H-burning limit and then are unable to sustain stable nuclear fusion of $\mathrm{H}$ like stars. However, unlike planets, BDs once formed go through a slow gravitational contraction process that releases potential energy and makes them shine. Thus, their brightness decreases with time as the energy reservoir diminishes and they

Send offprint requests to: $\mathrm{C}$. Saffe,

e-mail: saffe@oac.uncor.edu

* Based on observations collected at the European Southern Observatory, Chile, (ESO proposal 67.C-00365).

$\star \star$ On a fellowship from CONICET, Argentina.

$\star \star \star$ Visiting Astronomer, Complejo Astronómico el Leoncito operated under agreement between the Consejo Nacional de Investigaciones Científicas y Técnicas de la República Argentina and the National Universities of La Plata, Córdoba, and San Juan. cool down. Following Oppenheimer et al. (2000) we adopt $80 M_{J}\left(1 M_{J}=10^{-3} M_{\odot}\right)$ as the upper mass limit for BDs and $13 M_{J}$ as the lower mass limit. Solar metallicity objects with masses within these limits can burn deuterium at least during part of the evolution (Burrows et al. 1997). Below $13 M_{J}$ there is no nuclear fusion of any element.

Several investigations suggest a star-like formation mode for BDs (see, for example, Elmegreen 1999; Natta \& Testi 2001). However other two scenarios, ejection of stellar embryos (Reipurth \& Clarke 2001) and planet-like formation in circumstellar disks (Pickett et al. 2000) have been proposed. The current observational evidence seems to provide support to the stellar-like formation process (see, for example, Muench et al. 2002), although neither the embryos ejection nor the planet-like formation hypothesis can presently be disregarded on observational grounds. Understanding the formation process of BDs is very important as it can shed light on the mechanism of formation of both stars and planets.

BDs are elusive because they are very faint. These objects are easier to detect when young since they are brighter and indeed several young BDs are known to date in nearby dark clouds (see, for example, Briceño et al. 1998; Comerón et al. 2000; Briceño et al. 2002). A complete census of these objects 
in a given star-forming region would allow us to carry out a meaningful estimate of their real number and thus a measurement of the IMF of the cloud. However a considerable observational effort is required to fully accomplish this aim.

In this contribution we present results of optical spectroscopic observations of eight candidate young BDs in the Chamaeleon I dark cloud proposed by several recent infrared surveys aimed at finding sub-stellar objects in this star-forming region (see, for example, Persi et al. 2000; Gómez \& Kenyon 2001). An optical spectrum of $\mathrm{Sz} \mathrm{33,} \mathrm{a} \mathrm{previously} \mathrm{known}$ $\mathrm{T}$ Tauri star of the cloud, is also presented. Our final goal is to carry out a precise determination of the complete IMF of the cloud.

In Sect. 2 we describe the observations and data reduction. In Sect. 3 we present our analysis and results. We derive spectral types from the $\mathrm{TiO}$ and $\mathrm{VO}$ indexes and the PC3 index of Martín et al. (1999) which combined with published photometry allows us to place the stars in the HR diagram. We conclude with a brief summary in Sect. 4 .

\section{Observations and data reduction}

The observations were carried out from May to July 2001 in service mode with FORS1 on the ESO-VLT UT1. This instrument has different configurations ${ }^{1}$. We used the MOS (multi-object spectroscopy) sub-mode in combination with the grisms GRISM-600R and GRISM-600I to cover the spectral range between 5300 and $9000 \AA$, approximately, on a $2048 \times 2048$ pixels CCD detector. These grisms provide resolving powers $R=1230$ and 1530, respectively. The corresponding spectral dispersions are 1.08 and $1.06 \AA /$ pix. Two order separation filters, GG435+31 and OG590+72, were employed with grisms GRISM-600R and GRISM-600I, respectively, to isolate the useful spectral range for each grism.

MOS has 19 movable slitlets that can be displaced by linear guides to any position along the dispersion direction in the field of view. In addition, the instrument can be rotated around its optical axis allowing a wide variety of target configurations. The width of each individual slitlet is also adjustable. For our observations we selected a width of $1^{\prime \prime}$ for all the 19 slitlets. Even-numbered slitlets are 20" long and oddnumbered are $22^{\prime \prime}$ long.

In addition to the VLT observations we obtained spectra of two relatively bright objects (ISO-ChaI 126 and ISOChaI 237, see Table 2) with the 2.15-m telescope at the CASLEO (San Juan, Argentina) in April 2000. We used the REOSC spectrograph in SD (simple dispersion) mode with a $300 \mathrm{l} / \mathrm{mm}$ grating blazed at $5000 \AA$ and a long slit opened to $\sim 2^{\prime \prime}$. We registered the spectral range between 4500 and $8500 \AA$ on a $1024 \times 1024$ thinned TEK CCD detector at a dispersion of $\sim 4 \AA /$ pix, resulting in a resolving power $R=750$.

We reduced the data using standard IRAF procedures ${ }^{2}$. We trimmed the CCD frames at each end of the slit, corrected

\footnotetext{
${ }^{1}$ See the FORS1+2 User Manual for a full description on the different observing modes of this instrument, available at: http://www. eso.org/instruments/fors1/

2 IRAF is distributed by the National Optical Astronomy Observatory, which is operated by the Association of Universities for
}

for the bias level and divided by appropriate normalized flatfields. The spectra were extracted using the NOAO task apextract with an aperture of 5 pixel radius. A sky subtraction was done by fitting a polynomial to the regions on either side of the aperture. Residual night sky lines were removed by interpolating across them. A non linear low order fit to the lines in the HeNeAr lamp (VLT spectra) or CuNeAr lamp (CASLEO data) was used to wavelength calibrate the spectra. Typical RMS for the wavelength solutions are 0.07 and 0.04 for the grisms GRISM-600R and GRISM-600I, respectively. We obtained values of $\sim 0.5$ for the RMS corresponding to the CASLEO solution. The VLT-FORS spectra have been flux-calibrated and normalized to the flux at $6800 \AA$. CASLEO data are presented in count units as no flux standards were observed in this case.

\section{Data analysis and results}

\subsection{The sample}

In Table 1 we list our sample stars together with their coordinates and observing logs; in Table 2 photometric data from the literature are provided. Seven of the nine stars in our sample were selected among young low mass and brown dwarf candidates proposed by several recent infrared surveys of the cloud (Cambrésy et al. 1998; Persi et al. 2000; Gómez \& Kenyon 2001; Kenyon \& Gómez 2001). These objects, unlike most of the proposed candidates, have optical counterparts on the the Digitized Sky Survey ${ }^{3}$ (DSS) plates and thus optical spectra can be obtained. The existence of an optical object at the position of the infrared discovered source was the selection criterion used to define the present sample.

[SGR2003] 1 is the only object not selected as a candidate very low mass member of the cloud by previous surveys. This object was observed simultaneously with [GK2001] 30 (ISO-ChaI 220). The central slit of the MOS was placed on [GK2001] 30 whereas the rest of the slits on optical stars in the field. One of these objects (identified as [SGR2003] 1) turned out to be a new low mass cloud member judging from the strong emission in $\mathrm{H} \alpha$ (equivalent width $=-182.6$ ). Table 1 provides the coordinates of this object and Table $2 \mathrm{~K}$ and $H$ magnitudes (Gómez \& Kenyon 2001). [SGR2003] 1 was not detected at $J$ and thus not placed on the color-color $(J-H$ vs. $H-K$ ) diagram to determine likely near-infrared color excesses. Similarly Sz 33, a previously known T Tauri star of the cloud (see, for example, Lawson et al. 1996)

Research in Astronomy, Inc. under contract to the National Science Foundation.

${ }^{3}$ Based on photographic data obtained using The UK Schmidt Telescope. The UK Schmidt Telescope was operated by the Royal Observatory Edinburgh, with funding from the UK Science and Engineering Research Council, until 1988 June, and thereafter by the Anglo-Australian Observatory. Original plate material is copyright (c) the Royal Observatory Edinburgh and the Anglo-Australian Observatory. The plates were processed into the present compressed digital form with their permission. The Digitized Sky Survey was produced at the Space Telescope Science Institute under US Government grant NAG W-2166. Copyright (c) 1993, 1994, Association of Universities of Research in Astronomy, Inc. All right reserved. 
Table 1. Observed objects.

\begin{tabular}{|c|c|c|c|c|c|c|}
\hline Name & $\begin{array}{l}\alpha(2000.0) \\
{[\mathrm{h}: \mathrm{m}: \mathrm{s}]}\end{array}$ & $\begin{array}{l}\delta(2000.0) \\
{\left[{ }^{o}:^{\prime}:^{\prime \prime}\right]} \\
\end{array}$ & $\begin{array}{r}I T R \\
{[\mathrm{~s}]} \\
\end{array}$ & $\begin{array}{r}I T I \\
{[\mathrm{~s}]} \\
\end{array}$ & Tel+Instru & Other ID \\
\hline [GK2001] 8 & 110548.8 & -764017 & 3050 & 2950 & VLT+MOS & \\
\hline ISO-ChaI 126 & 110804.2 & -773843 & 900 & & CASLEO+REOSC & [CCE98] 32 \\
\hline [KG2001] 102 & 110949.3 & -773120 & $2 \times 3105$ & 2960 & VLT+MOS & \\
\hline [SGR2003] 1 & 110953.1 & -773058 & $2 \times 3105$ & 2960 & VLT+MOS & \\
\hline [GK2001] 30 & 110953.4 & -772836 & $2 \times 3105$ & 2960 & VLT+MOS & ISO-ChaI 220 \\
\hline $\mathrm{Sz} 33$ & 110953.9 & -762925 & 3040 & 2960 & VLT+MOS & ISO-ChaI 224, [KG2001] 110 \\
\hline [GK2001] 31 & 110955.0 & -763112 & 3040 & 2960 & VLT+MOS & ISO-ChaI 225 \\
\hline ISO-ChaI 237 & 111011.9 & -773531 & 900 & & CASLEO+REOSC & [CCE98] 48, [OTS99] 45, [KG2001] 121 \\
\hline [GK2001] 53 & 111423.6 & -775612 & 3300 & 3300 & $\mathrm{VLT}+\mathrm{MOS}$ & \\
\hline
\end{tabular}

Note: We adopt the SIMBAD source denomination scheme which is based on the recommendations of the IAU Commission 5 Task Group on Designations.

Table 2. Compiled photometric data and $\mathrm{H} \alpha$ equivalent width measurements.

\begin{tabular}{|c|c|c|c|c|c|c|c|}
\hline Name & $L^{\mathrm{a}}$ & $K^{\mathrm{b}}$ & $H^{\mathrm{b}}$ & $J^{\mathrm{b}}$ & $I^{\mathrm{c}}$ & $\mathrm{H} \alpha(E W[\AA])$ & Na I 8183-8195 (EW[Å]) \\
\hline [GK2001] 8 & & 13.94 & 14.67 & 15.22 & & $<-5$ & 4.9 \\
\hline ISO-ChaI 126 & & 8.30 & 9.67 & 11.51 & 14.39 & -97.1 & \\
\hline [KG2001] 102 & 10.62 & 11.07 & 11.45 & 12.47 & & -87.2 & 2.7 \\
\hline [SGR2003] 1 & & 14.87 & 15.64 & & & -182.6 & \\
\hline [GK2001] 30 & & 12.48 & 13.33 & 14.57 & 18.11 & -92.3 & 2.1 \\
\hline Sz 33 & 8.22 & 9.10 & 9.84 & 11.19 & 13.99 & -9.6 & 2.2 \\
\hline [GK2001] 31 & & 12.75 & 13.73 & 14.84 & 17.12 & -68.7 & 1.8 \\
\hline ISO-ChaI 237 & 7.69 & 8.55 & 9.34 & 10.75 & 13.90 & $<-3$ & \\
\hline [GK2001] 53 & & 13.73 & 14.57 & & & -15.7 & 6.2 \\
\hline
\end{tabular}

\footnotetext{
${ }^{a}$ Kenyon \& Gómez (2001).

b Gómez \& Kenyon (2001).

${ }^{\mathrm{c}}$ Cambrésy et al. (1998).
}

lying in the close vicinity of [GK2001] 31 (ISO-ChaI 225), was observed on the same MOS frame as this object.

\subsection{The spectra}

We mention that for five objects in our sample (ISO-ChaI 126, [GK2001] 30, Sz 33, [GK2001] 31, and ISO-ChaI 237) infrared spectra are available in the literature (Gómez \& Persi 2002; Gómez \& Mardones 2003). However most of these spectra are almost featureless at the spectral resolution used, thus not allowing a full characterization of the objects. [GK2001] 30 shows $\mathrm{Br} \gamma$ in emission while $\mathrm{Sz} 33$ have $\mathrm{Pa} \beta, \mathrm{Pa} \delta$ and $\mathrm{Br} \gamma$ in emission. The spectral slope of these sources, with exception of $\mathrm{Sz} 33$, increases with the wavelength suggesting the presence of cool material probably in a circumstellar disk. Optical low resolution spectra of these objects provide additional useful information to support their pre-main sequence status. In particular $\mathrm{H} \alpha$ in emission is a distinctive feature of T Tauri stars and of previously known young BDs. Furthermore, spectral classification schemes based on optical features are in general better developed than near-infrared systems and thus can provide more reliable estimates for the low-mass candidates.

Figures 1-5 show the spectra of the observed objects. Photospheric features such as molecular bands of $\mathrm{CaH}$, $\mathrm{TiO}$, and $\mathrm{VO}$, as well as atomic lines of $\mathrm{Na} \mathrm{I}$ and $\mathrm{K} \mathrm{I}$ are indicated. These molecular and atomic features are common among M-type type stars (Kirkpatrick et al. 1991).

In the following sections we will use the detection of specific spectral features (such as $\mathrm{H} \alpha$ in emission and the $\mathrm{Na} \mathrm{I}$ absorption doublet) to unveil the nature of our sample objects. The strength of the TiO molecular bands at 6180 and $7100 \AA$ and the PC3 index of Martín et al. (1999) will be applied to derive spectral types for these objects.

\subsubsection{Spectral features}

Table 2 lists $\mathrm{H} \alpha$ equivalent width measurements for the observed objects. We estimate an uncertainty of the order of 3-5\% due to noise in our spectra and errors in placing the continuum level. Five objects in our sample (ISO-ChaI 126, [KG2001] 102, [SGR2003] 1, [GK2001] 30 and [GK2001] 31) show conspicuous $\mathrm{H} \alpha$ emission line with equivalent widths $>50 \AA . \mathrm{Sz} 33$ and [GK2001] 53 have moderate emission in this line. For other two objects in the sample, [GK2001]8 and ISO-ChaI 237, we do not see a strong $\mathrm{H} \alpha$ in emission and are able to measure only an upper limit to its equivalent widths. Whereas higher signal to noise and resolution spectra are required to check in detail for the presence (absence) of $\mathrm{H} \alpha$ in emission, these two objects are most likely foreground dwarfs or background giants. As a group, spectra in Figs. 1-5 show similar characteristics as 

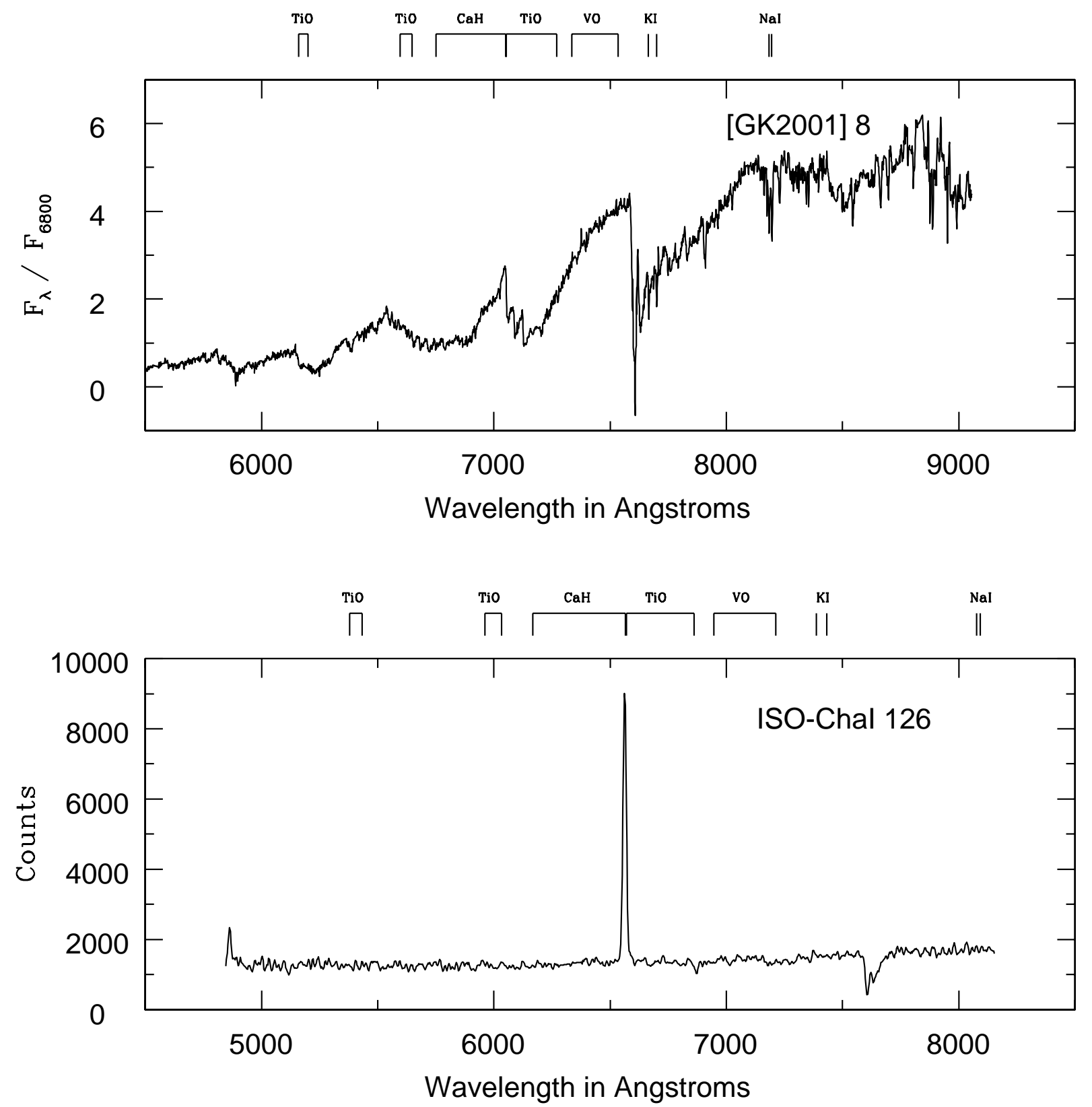

Fig. 1. Optical spectra of [GK2001] 8 and ISO-ChaI 126 obtained with the VLT+MOS and the CASLEO+REOSC, respectively. Molecular and atomic features common in M-type stars are indicated. The [GK2001] 8 spectrum has been flux-calibrated and normalized to the flux at $6800 \AA$ A.

M-type T Tauri stars and previously know young BDs in star forming regions (see, for example, Schwartz 1977; Briceño et al. 1998; Comerón et al. 2000; Martín et al. 2001). In particular, we note that the equivalent widths of $\mathrm{H} \alpha$ are comparable with those measured by Comerón et al. (2000) for their sample of very low mass stars and BDs in Chamaeleon I.

Martín et al. (1996) in their study of BDs in the Pleiades found that, due to lower gravity, the $\mathrm{Na}$ I doublet (8183-8195 $\AA$ ) tends to be weaker in young sub-stellar objects than in field stars of identical spectral type. These authors analyzed objects with spectral types in the M 3.5-M 9.5 range. Table 2 lists the Na I doublet equivalent widths for the objects in our sample for which the red part of the spectrum is available and of good quality. We estimate an uncertainty of $\sim 0.5 \AA$ in our measurements. Using the spectral types derived in the next section (see Table 3), we compare the Na I doublet equivalent widths of our objects with those of Pleiades and field stars listed by Martín et al. (1996, their Table 5).

Three of the objects ([KG2001] 102, [GK2001] 30 and [GK2001] 31) have very modest $\mathrm{Na}$ I doublet equivalent widths, smaller than those of Pleiades and field stars of the same spectral type, confirming that they are very low mass young objects. The $\mathrm{Na}$ I doublet equivalent width of [GK2001] 53 is comparable to those of two Pleiades BDs of the same spectral type, while the equivalent width of [GK2001] 8 is larger than that of the Pleiades BD Calar 4 (with identical spectral type as [GK2001] 8) and comparable to that of field dwarfs. This supports the hypothesis that [GK2001] 8 is not a 

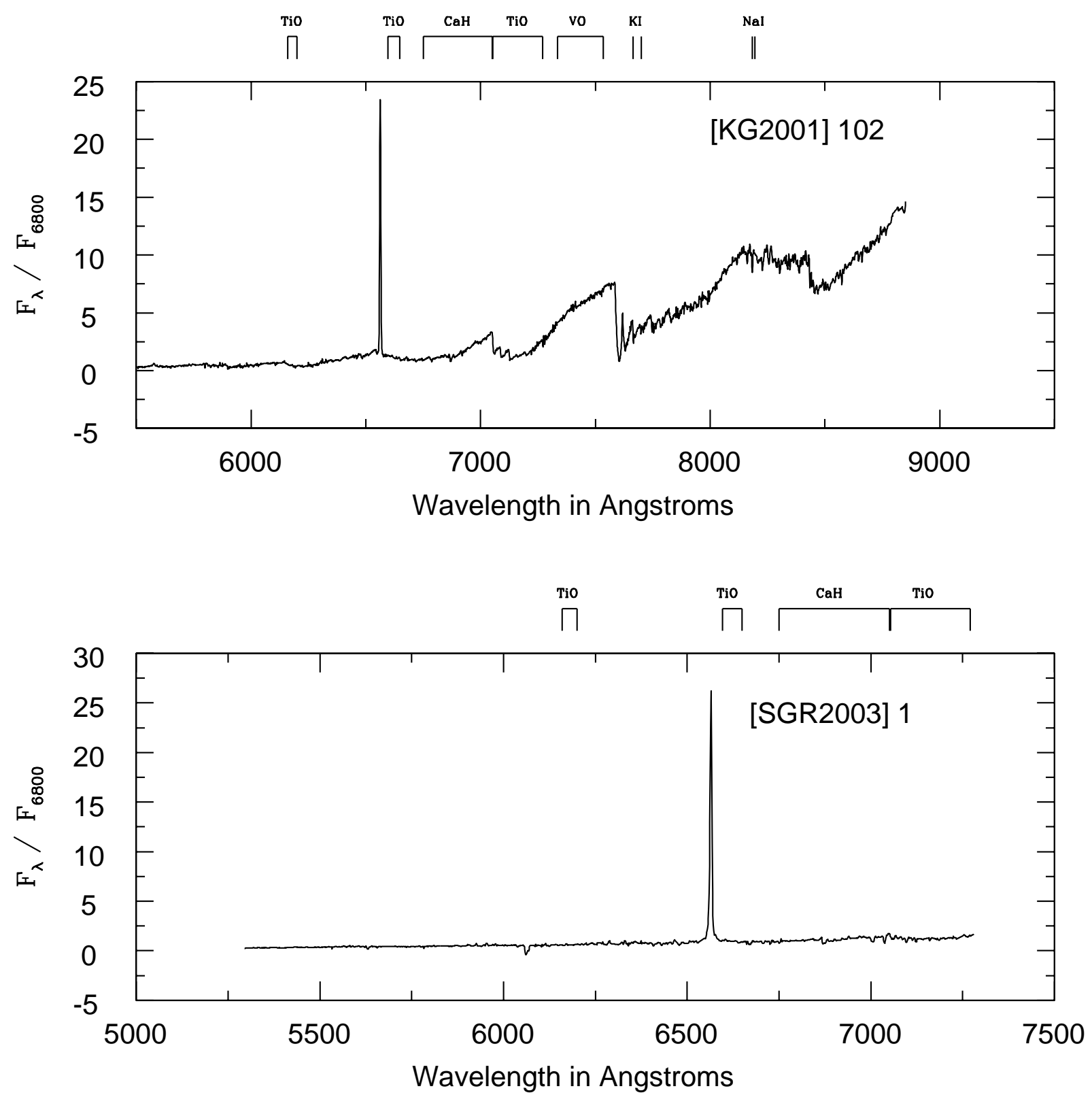

Fig. 2. Optical spectra of [KS2001] 102 and [SGR2003] 1 obtained with the VLT+MOS. Molecular and atomic features common in M-type stars are indicated. The spectra are flux-calibrated and normalized to the flux at $6800 \AA$.

low mass member of Chamaeleon I and is likely a foreground dwarf. Finally, Sz 33 with a M 2 spectral type is outside the range analyzed by Martín et al. (1996).

Finally, whereas the Li I $\lambda 6707.8$ line is a well-known indicator of youth in late-type fully convective stars (see, for example, Magazzù et al. 1993), the modest spectral resolution of our spectra, in combination with the low $\mathrm{S} / \mathrm{N}$ ratio in a few cases, prevented us from detecting this line in our sample objects. Higher resolution data are thus required to unambiguously detect this line in the spectra of the present sample.

In summary, $\mathrm{H} \alpha$ together with the $\mathrm{Na}$ I doublet confirm that [KG2001]102, [GK2001] 30 and [GK2001] 31 are very likely pre-main sequence, low mass cloud members, while the spectra of [GK2001] 8 and ISO-ChaI 237 do not support cloud membership; the status of [GK2001] 53 remains uncertain, since it has an $\mathrm{H} \alpha$ comparable to that of previously known low mass cloud members, but a somewhat high $\mathrm{Na}$ I doublet equivalent width. However the equivalent width of this doublet for [GK2001] 53 is still comparable to those of two Pleiades BDs of the same spectral type. Finally, whereas we were not able to use the Na I doublet for Sz 33, ISO-ChaI 126, and [SGR2003] 1, the presence of $\mathrm{H} \alpha$ in emission in their spectra supports the pre-main sequence nature of these sources. In addition $\mathrm{Sz} 33$ is a previously known T Tauri member of the cloud (Lawson et al. 1996).

\subsubsection{Spectral types}

In order to derive spectral types for our sources, we used the two TiO absorption bands at 6180 and $7100 \AA$ that are temperature sensitive for M dwarfs and giants (O'Connell 1973). 

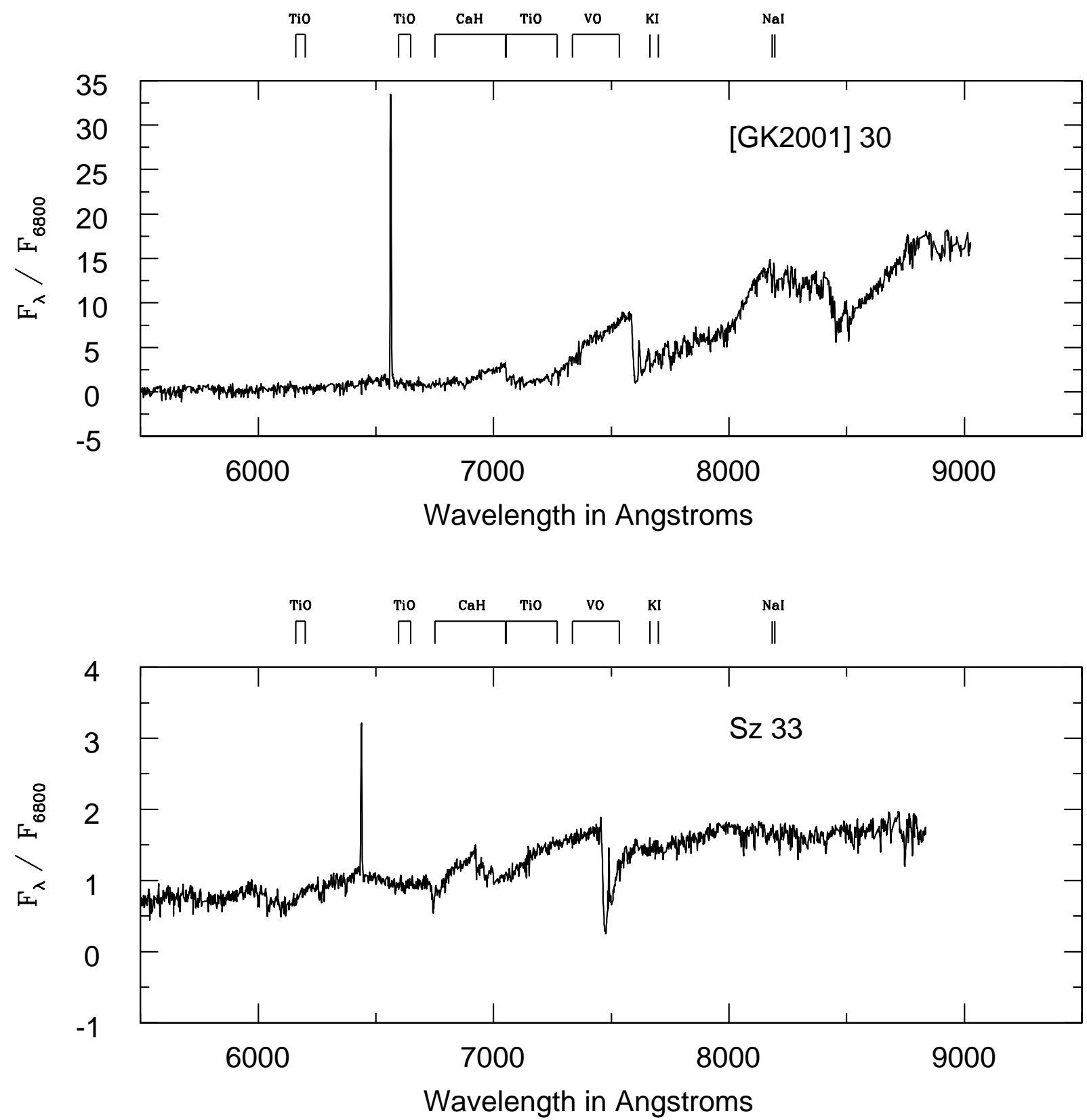

Fig. 3. Optical spectra of [GK2001] 30 and Sz 33 obtained with the VLT+MOS. Molecular and atomic features common in M-type stars are indicated. The spectra are flux-calibrated and normalized to the flux at $6800 \AA$.

We also applied the PC3 index form Martín et al. (1999). For stars with available flux-calibrated FORS spectra, we compared them with the detailed grid of late type standard stars obtained by Kirkpatrick et al. (1991), Henry et al. (1994), and Kirkpatrick et al. (1995).

Kenyon et al. (1998) applied the Titanium Oxide band indexes at 6180 and $7100 \AA$ to estimate spectral types for pre-main sequence stars in the Taurus-Auriga molecular cloud. Each index measures the depth of the $\mathrm{TiO}$ band at a given wavelength relative to an interpolated nearby continuum point. These authors defined $[\mathrm{TiO}]_{1}$ and $[\mathrm{TiO}]_{2}$ as:

$$
[\mathrm{TiO}]_{1}=-2.5 \log \left[\frac{F_{6180}}{F_{6125}+0.225\left(F_{6370}-F_{6125}\right)}\right],
$$

$[\mathrm{TiO}]_{2}=-2.5 \log \left[\frac{F_{7100}}{F_{7025}+0.2\left(F_{7400}-F_{7025}\right)}\right]$

(see also O'Connell 1973). The $30 \AA$ bandpass filters used in these indexes are free from strong emission lines (such as $\mathrm{H} \alpha$ ) or telluric absorptions. In Fig. 6 we plot these indexes for our sources along with the dwarf and giant sequences from O'Connell (1973). Three objects (ISO-ChaI 126, [SGR2003] 1 and [GK2001] 30) are not shown in the figure due to the large veiling effect that mainly affects the $[\mathrm{TiO}]_{1}$ index as we discuss below.

The new objects, on average, lie slightly above the sequences defined by the dwarfs and giants, having weak $\left[\mathrm{TiO}_{1}\right.$ index relatively to the $\left[\mathrm{TiO}_{2}\right.$ absorption band. Kenyon et al. (1998) noticed the same tendency in many T Tauri stars with 

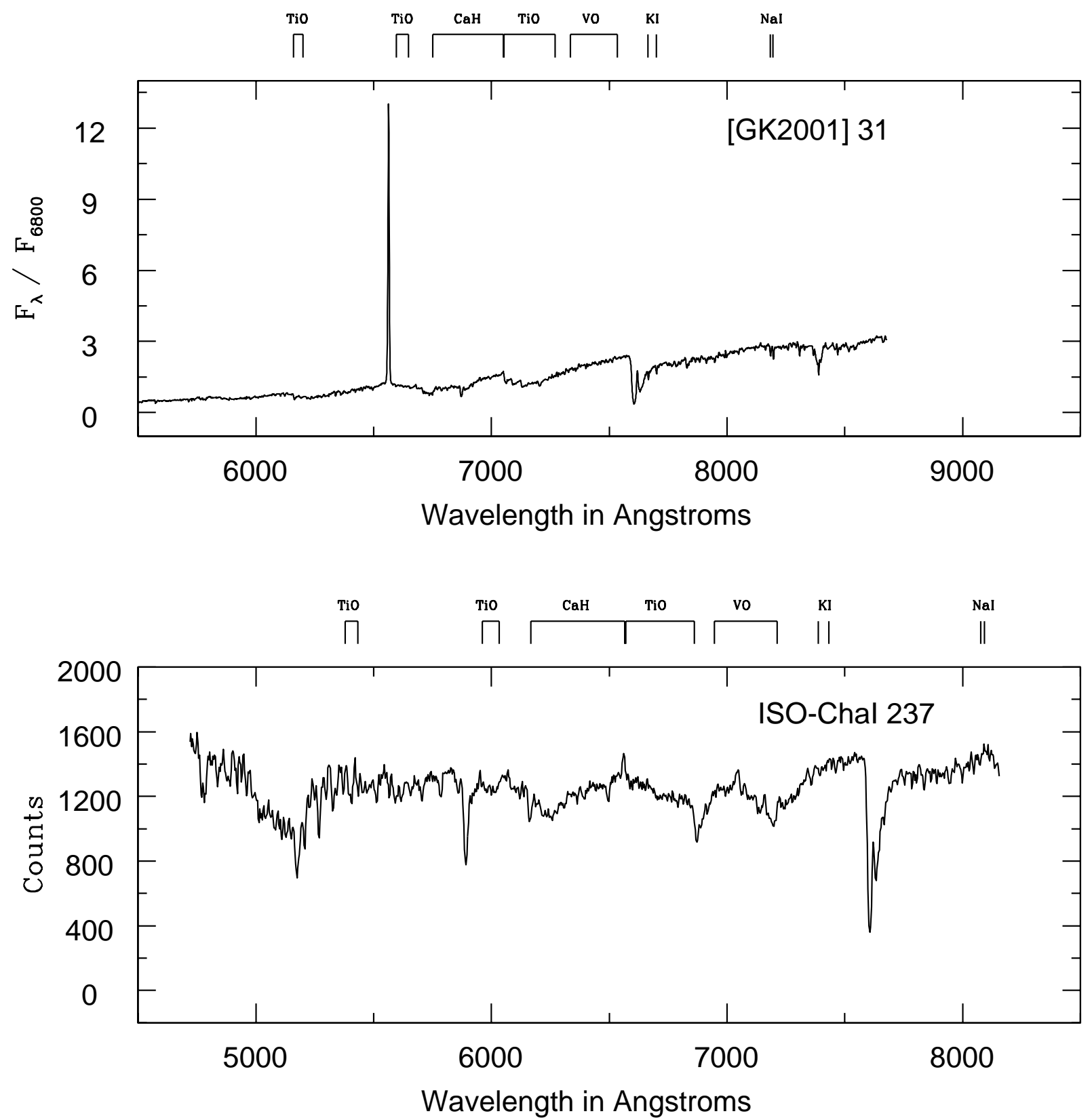

Fig. 4. Optical spectra of [GK2001] 31 and ISO-ChaI 237 obtained with the VLT+MOS and the CASLEO+REOSC, respectively. Molecular and atomic features common in M-type stars are indicated. The [GK2001] 31 spectrum has been flux-calibrated and normalized to the flux at $6800 \AA$.

strong emission lines in the Taurus cloud. The emission from a hot blue continuum source (with $T \sim 10^{4}$; Hartigan et al. 1991) may diminish the depth of the TiO bands and induce us to assign earlier spectral types to the central star. This veiling effect increases toward shorter (bluer) wavelengths, affecting more the band at $6180 \AA$ than the index at $7100 \AA$. As mentioned, five of the stars in Table 2 have $\mathrm{H} \alpha$ equivalent widths $>50 \AA$ which suggests the presence of veiling in their spectra.

As to the luminosity class, pre-main sequence stars are known to have surface gravities intermediate between those of dwarfs (luminosity class V) and giants (luminosity class III). Kirkpatrick et al. (1991) identified several features sensitive to the gravity of $M$ type stars. In particular the intensity of the $\mathrm{CaH}$ absorption band at $6975 \AA$ and the $\mathrm{Na} \mathrm{I}$ lines at 8183-8195 $\AA$ decrease with decreasing gravity and then are stronger in dwarfs than in giants. They defined two color ratios (A and C, respectively) that compare the fluxes in a $30 \AA$ band centered on these features with respect to a nearby continuum integrated over an identical bandpass. These color ratios for our sample give values intermediate between those of dwarfs and giants and, on average, slightly closer to the giant branch. We caution that the veiling effect may also affect the depth of the gravity sensitive features and hence lead us to derive lower gravities, closer to class III luminosity objects.

Three objects need to be discussed in detail. ISO-ChaI 126 shows a featureless spectrum (except for $\mathrm{H} \alpha$ and $\mathrm{H} \beta$, both in emission, see Fig. 1, lower panel); for this object we adopted a 


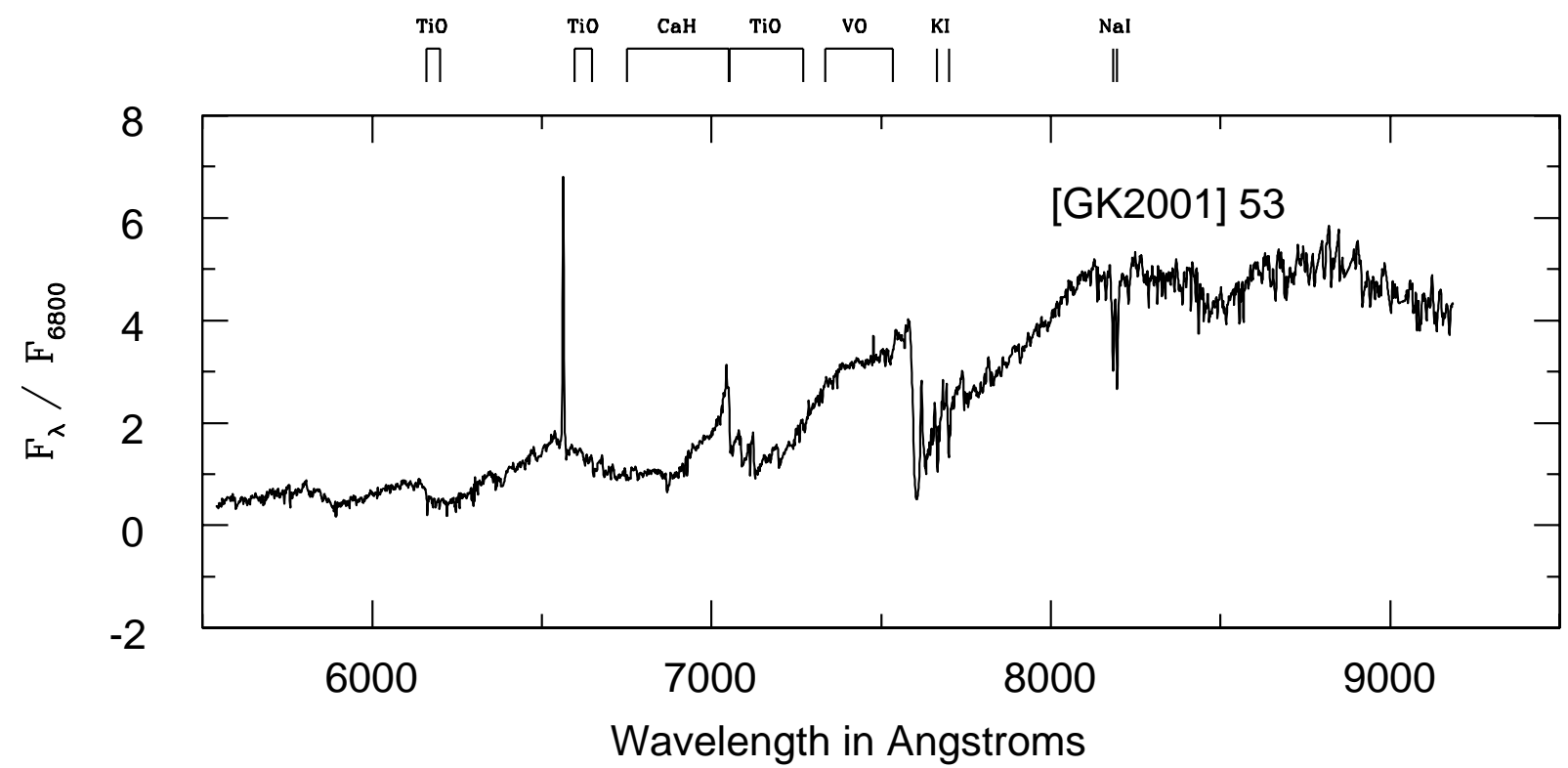

Fig. 5. Optical spectrum of [GK2001] 53 obtained with the VLT+MOS. Molecular and atomic features common in M-type stars are indicated. The spectrum has been flux-calibrated and normalized to the flux at $6800 \AA$.

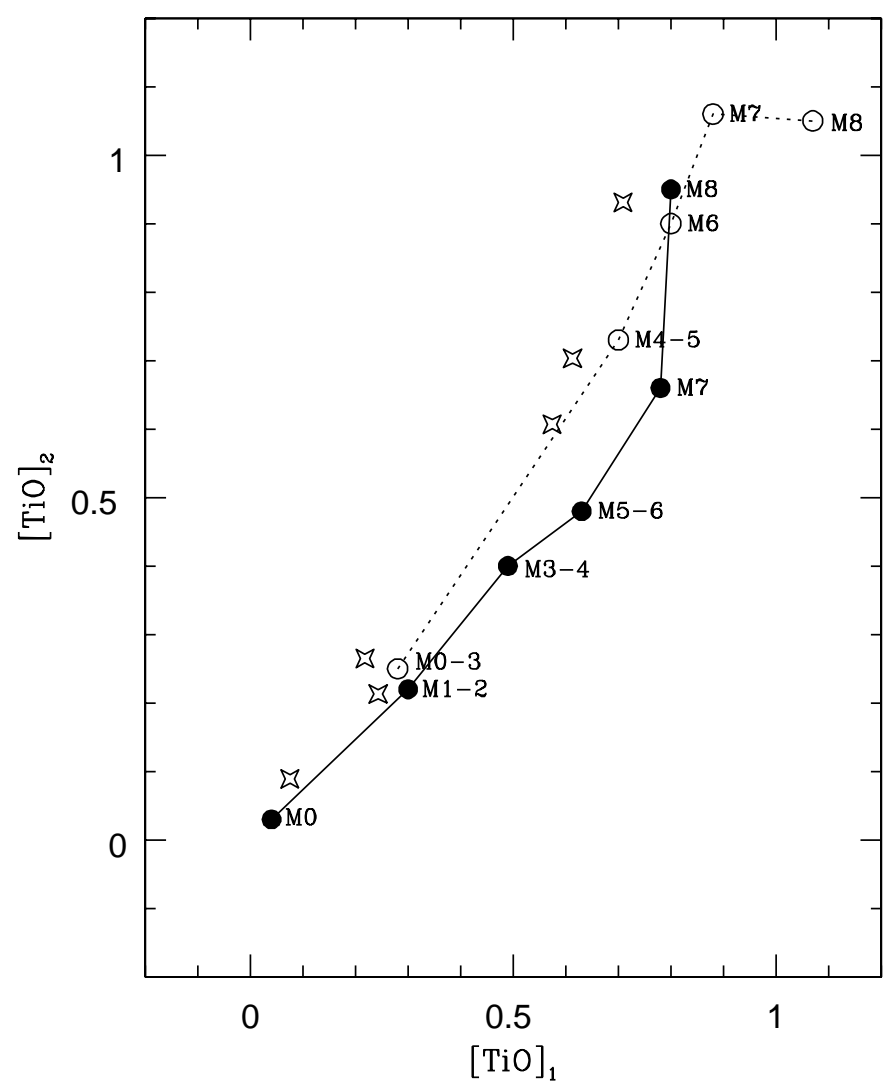

Fig. 6. $[\mathrm{TiO}]_{2}$ vs. $[\mathrm{TiO}]_{1}$ indexes for field dwarfs (filled circles) and giants (open circles) obtained from O'Connell (1973). The continuous line connects the points defining the dwarf sequence and the dashed line those corresponding to the giant branch. Six of the young stellar objects in Tables 1 and 2 are indicated with starred symbols.

M2 spectral type derived by Gómez \& Mardones (2003) from a near-infrared spectrum.
The $R$-band spectrum of [SGR2003] 1 has a strong H $\alpha$ line in emission and no other spectral feature and the $\mathrm{TiO}$ band at $6180 \AA$ is completely veiled (see Fig. 2 lower panel). The $I$-band region (not shown in Fig. 2 lower panel) was observed under poor sky conditions and some night sky lines were registered on the stellar spectrum. However, it clearly shows the TiO absorption at $7100 \AA$, with a depth corresponding to a M 6 spectral type. Due to the veiling effect, it is likely that the depth of this band is diminished and hence, as discussed, we are assigning a spectral type earlier than the actual type. The $I$-band region also shows the VO band centered at $7445 \AA$ that appears for spectral types as late as M 7 or later (Kirkpatrick et al. 1995); for the latest M-subtypes the VO band rather than the $\mathrm{TiO}$ band is indeed a more reliable indicator of temperature as the TiO starts to saturate (Kirkpatrick et al. 1991). Kirkpatrick et al. (1995) defined the VO ratio as:

VO ratio $=\frac{0.5625\left(F_{7350}-F_{7400}\right)+0.4375\left(F_{7510}-F_{7560}\right)}{F_{7420}-F_{7470}}$,

where $F_{\lambda_{1}}-F_{\lambda_{2}}$ indicates the flux integrated between $\lambda_{1}$ and $\lambda_{2}$. For M 7 stars they obtain $1.02 \leq \mathrm{VO}$ ratio $\leq 1.07$ and $1.07 \leq$ $\mathrm{VO}$ ratio $\leq 1.12$ for $\mathrm{M} 8$ stars. We derive $\mathrm{VO}$ ratio $=1.08$ for [SGR2003] 1 indicating an M 7-8 spectral type. We adopt an M 7 spectral type for this object; we caution however that we measured the VO band in a spectrum with sky emission lines superimposed on the stellar continuum. Although, in first approximation, this has a negligible effect on the depth of the detected absorption bands, better quality data are required to confirm our initial VO ratio measurement. We mention that a spectral type earlier than M 7 would shift horizontally to the left this object on the HR diagram (see Fig. 7), corresponding to larger values of mass and age. In particular for an M 6 spectral type we would obtain an age $>10^{7} \mathrm{yr}$, which is hard to conciliate with the strong $\mathrm{H} \alpha$ emission and highly veiled spectrum of this object. Both features indicate that the object is 


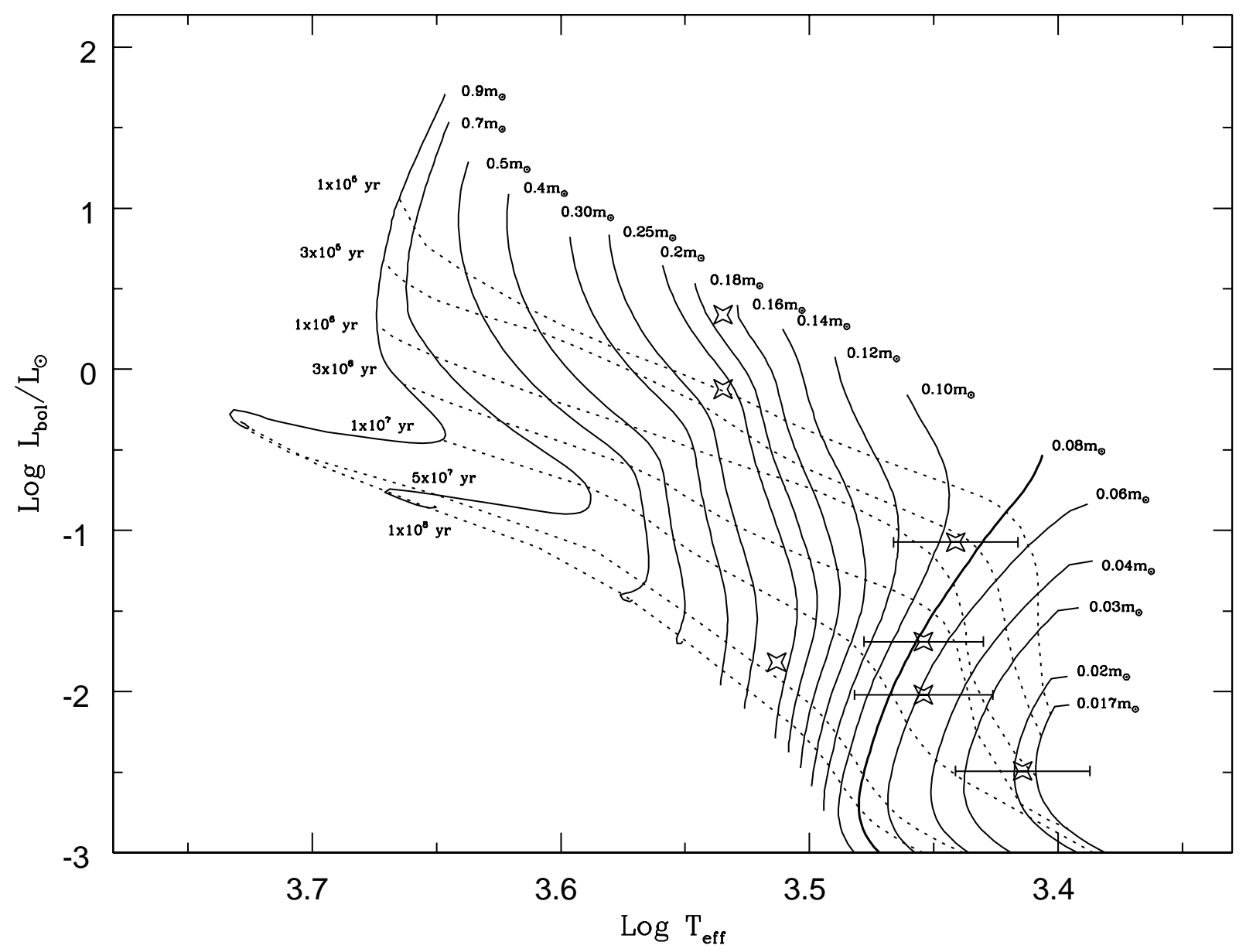

Fig. 7. HR diagram showing the positions of the seven of the observed stars (starred symbols). Pre-main sequence evolutionary tracks, indicated with continuous lines, are from D’Antona \& Mazzitelli (1997). The thick continuous line corresponds to $0.08 M_{\odot}\left(\sim 80 M_{\mathrm{J}}\right)$, the H burning limit. The dashed lines correspond to the isochrones calculated also by the same authors. The horizontal error bars correspond to a \pm 1 subtype uncertainty in the assigned spectral types.

most likely very young and probably not older than a few million years. In addition, the comparison of the $I$-band spectrum of [SGR2003] 1 with the grid of spectra of Kirkpatrick et al. (1991) indicates a spectral type M 7 for this source.

In the case of [GK2001] 30 strong sky lines contaminated the region around the $[\mathrm{TiO}]_{1}$ band and prevented us from measuring this index. In addition the $R$-band data are featureless (except for the presence of $\mathrm{H} \alpha$ in emission) indicating an increasing veiling effect towards this spectral region. From the depth of the TiO band at $7100 \AA$ we estimate a spectral type M 5 for this object.

Martín et al. (1999) used the spectral index PC3 to derive spectral types for late- $\mathrm{M}$ and $\mathrm{L}$ field dwarfs. This index compares the fluxes in two $40 \AA$ bands centered at 8250 and $7560 \AA$, respectively. For spectral types M 2.5-L1 they obtained the following relation:

$S p T=-6.685+11.715 \times(\mathrm{PC} 3)-2.024 \times(\mathrm{PC} 3)^{2}$.

We calculated this index for our sample objects and used Martín et al. (1999) calibration to estimate spectral types.

Table 3 lists the spectral types base on the TiO-band indexes corresponding to both the dwarf (left side) and giant (right side) sequences, as well as those obtained from the index PC3 from
Martín et al. (1999) and those derived from the comparison with the Kirkpatrick et al. (1991)'s grid. We also indicate the adopted spectral type for each object. For ISO-ChaI 126 the near-infrared spectrum derived spectral type is given. In the case of [SGR2003] 1 we used the VO band rather than the TiO band to estimate spectral type as mentioned above. We estimate an uncertainty of about \pm 1 subclass for the derived spectral types based on measurement errors of the $\mathrm{TiO}$ and $\mathrm{VO}$ bands. The same uncertainty is expected from spectral types derived by the comparison with Kirkpatrick et al. (1991)'s grid and from the PC3 index of Martín et al. (1999). The veiling effect may increase this uncertainty up to \pm 2 for the highly veiled and mostly featureless objects.

As already mentioned, five of the objects (ISO-ChaI 126, [GK2001] 30, Sz 33, [GK2001] 31, and ISO-ChaI 237) have near-infrared spectra published and derived spectral types and masses (Gómez \& Mardones 2003; Gómez \& Persi 2002). Excluding ISO-ChaI 126 for which we adopted the nearinfrared spectral type, for [GK2001] 30, ISO-ChaI 237, and [GK2001] 31, the optical and near-infrared derived spectral types agree within \pm 1 subtype. For $\mathrm{Sz} 33$ we instead estimated a M 0 spectral type from our optical spectrum, while 
Table 3. Derived parameters for the observed objects.

\begin{tabular}{|c|c|c|c|c|c|c|c|c|c|}
\hline Name & $\mathrm{S} . \mathrm{T}(\mathrm{TiO})^{\mathrm{a}}$ & S.T(PC3) ${ }^{\mathrm{b}}$ & S.T(K91 grid) ${ }^{\mathrm{c}}$ & S.T(adopted) & $T_{\mathrm{eff}}^{\mathrm{e}}$ & $A_{J}{ }^{\mathrm{f}}$ & $\log L_{\mathrm{bol}} / L_{\odot}$ & $M / M_{\odot}$ & Age [yr] \\
\hline [GK2001] 8 & $\mathrm{M} 7 / \mathrm{M} 4$ & M 5 & M 4.5 & M 5 & & & & & \\
\hline ISO-ChaI 126 & & & & $\mathrm{M} 2^{\mathrm{d}}$ & 3426 & 3.4 & 0.34 & 0.17 & $<1 \times 10^{5}$ \\
\hline [KG2001] 102 & M 8/M 6 & M 6 & M 6.5 & M 6 & 2762 & 1.1 & -1.071 & 0.09 & $4 \times 10^{5}$ \\
\hline [SGR2003] 1 & & M 7 & M 7 & M 7 & 2592 & & $-2.49^{\mathrm{g}}$ & 0.019 & $2 \times 10^{6}$ \\
\hline [GK2001] 30 & & M 6 & M 5.5 & M 5.5 & 2845 & 1.7 & -1.69 & 0.075 & $4 \times 10^{6}$ \\
\hline $\mathrm{Sz} 33$ & M 2/M 2 & & M 2 & M 2 & 3426 & 1.8 & -0.12 & 0.22 & $1 \times 10^{5}$ \\
\hline [GK2001] 31 & M 2/M 3 & M 3.5 & M 3 & M 3 & 3260 & 1.4 & -1.81 & 0.22 & $4 \times 10^{7}$ \\
\hline ISO-ChaI 237 & M 0/M 0 & & & M 0 & & & & & \\
\hline [GK2001] 53 & M 6.5/M 5 & M 5.5 & M 5 & M 5.5 & 2845 & & $-2.02^{\mathrm{g}}$ & 0.06 & $6 \times 10^{6}$ \\
\hline
\end{tabular}

${ }^{a}$ Left and right sides from dwarf and giant branches, respectively, O'Connell (1973).

${ }^{\mathrm{b}}$ Estimated from Martín et al. (1999).

c Estimated from Kirkpatrick et al. (1991)'s grid.

d Gómez \& Mardones (2003).

e Obtained from Wilking et al. (1999) calibration.

${ }^{\mathrm{f}}$ We obtain $A_{K}=1.0$ and 0.895 for [SGR2003] 1 and [GK2001] 53, respectively.

g Derived using $K$ and $H$ magnitudes and $A_{K}=1.79 \mathrm{E}(H-K)$ obtained from Rieke \& Lebofsky (1985).

Gómez \& Persi (2002) obtained a M 2 type from the measured near-infrared water vapor bands.

In order to derive effective temperatures, intrinsic colors, and bolometric corrections for our targets from the estimated spectral types, we adopted the calibrations obtained by Wilking et al. (1999). These relations are valid for spectral types from M 2 to M 9.

\section{Luminosities, masses and ages}

We used the $J$ magnitudes to estimate bolometric luminosities. This spectral band is less affected by contamination from circumstellar infrared excess emission than the $K$ or $H$ data. The bolometric luminosities are calculated from the following expressions:

$\log \left(L_{\mathrm{bol}} / L_{\odot}\right)=1.89-0.4 M_{\mathrm{bol}}$

$M_{\mathrm{bol}}=J-A_{J}-D M+B C_{J}$

$A_{J}=2.63 E(J-H)$

where $D M=6.0$ is the distance modulus $(d=160 \mathrm{pc}$; Whittet et al. 1997), $B C_{J}$ is the $J$-band bolometric correction, and $J$ is the apparent magnitude. $A_{J}$ is the extinction derived from the Rieke \& Lebofsky (1985) reddening law, with $E(J-H)=(J-H)-(J-H)_{\mathrm{o}}$ the color excess, and $(J-H)_{\mathrm{o}}$ the intrinsic color. For two objects, [SGR2003] 1 and [GK2001] 53, with no $J$ magnitude measurements we used the $K$ band data in combination with the Rieke \& Lebofsky (1985) reddening law, $A_{K}=1.79 E(H-K)$ (with $E(H-K)=(H-K)-(H-K)_{\mathrm{o}}$ and $(H-K)_{\mathrm{o}}$ the intrinsic color) and the $K$-band bolometric correction, $B C_{K}$, to estimate the bolometric luminosities.

Luminosities for three of the objects (ISO-Cha I 126, [GK2001] 30, and [GK2001] 31) were previously determined by Persi et al. (2000). Our determinations agree with those of Persi et al. within a factor of $\sim 2$, with exception of ISO-Cha I 126. For this object, the main discrepancy comes from the estimated $A_{J}$. Persi et al. (2000) based their determination on the $(I-J)$ color obtained by Cambrésy et al. (1998) while here we use the $(J-H)$ color index from Gómez \& Kenyon (2001) to compute $A_{J}$ (cf. Tables 4 and 3 in Persi et al. 2000). Additional measurements of the $I$ and $H$ magnitudes may help to clarify this apparent lack of agreement.

We chose pre-main sequence evolutionary tracks and isochrones from D'Antona \& Mazzitelli $(1997)^{4}$ to derive masses and ages for the observed sources. Gómez \& Mardones (2003) used these models to study the mass and age distributions of the young stars belonging to the Chamaeleon I dark cloud. D'Antona \& Mazzitelli (1997)'s calculations provide consistent and plausible results for the group of objects they analyzed in reasonable agreement with the higher mass members of the cloud (see Lawson et al. 1996). Table 3 lists these parameters. Figure 7 shows the locations of observed Chamaeleon I stars on the HR diagram.

We excluded from our analysis [GK2001] 8 and ISOChaI 237 as they are probably not young cloud members based on the $\mathrm{H} \alpha$ emission and $\mathrm{Na}$ I intensity in the case of [GK2001] 8 (see Sect. 3.2.1.). Additional data and in particular higher resolution spectra around $\mathrm{H} \alpha$ are necessary to establish the true nature of these objects. On the other hand, we include [GK2001] 53 in this section although the $\mathrm{H} \alpha$ and $\mathrm{Na}$ I doublet equivalent width measurements do not allow us to unambiguously assert (disregard) the pre-main sequence nature of this object.

Four objects in our sample ([KG2001] 102, [GK2001] 30, [SGR2003] 1, and [GK2001] 53) have masses close or below the H-burning limit (i.e., $0.08 M_{\odot}, \sim 80 M_{J}$ ). In Fig. 7, the horizontal error bars indicate the displacements on the HR diagram that these objects would experiment due to \pm 1 sub-type (i.e.,

\footnotetext{
${ }^{4}$ Available at

http://www.mporzio.astro.it/ dantona/prems.html
} 


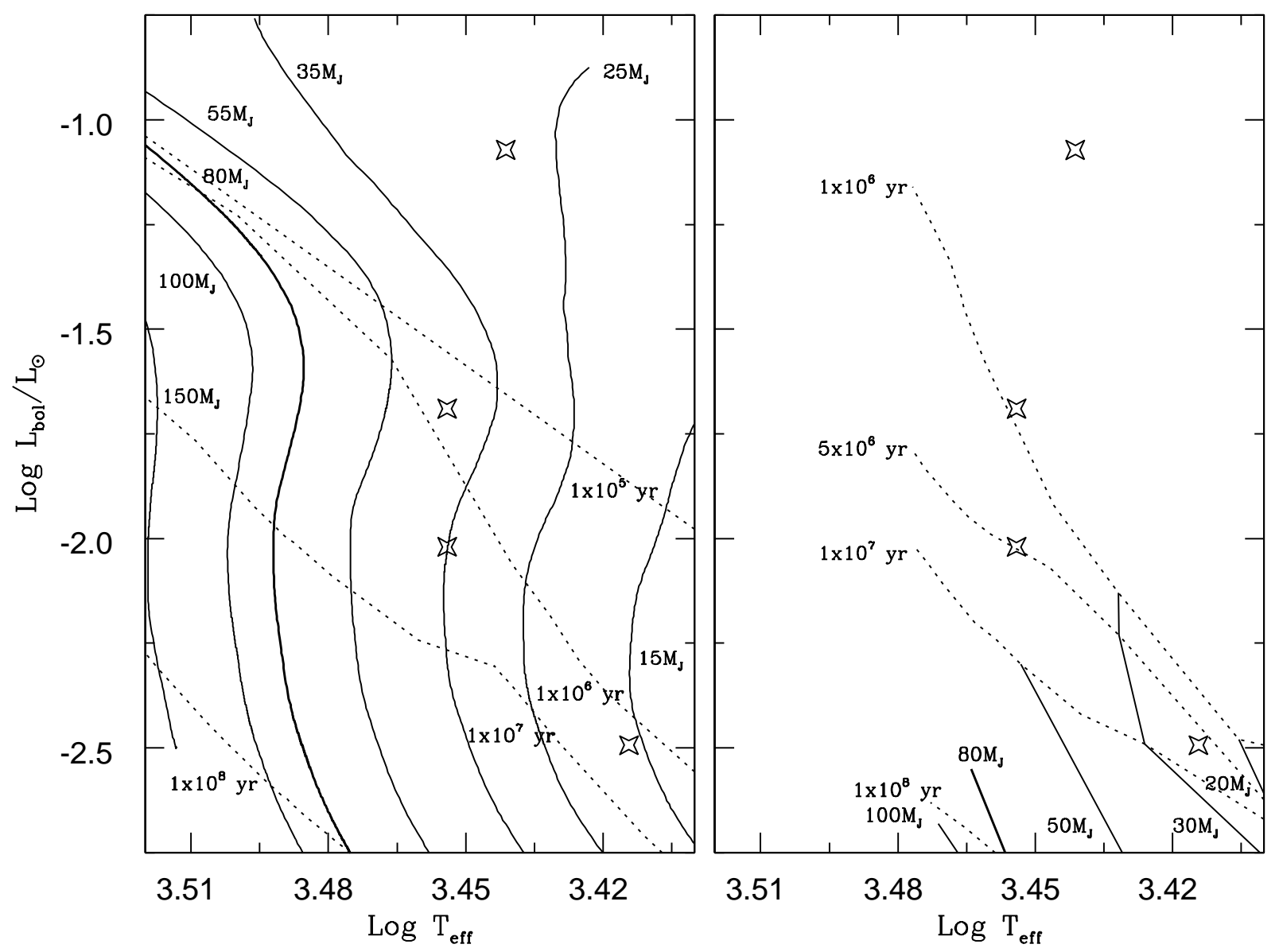

Fig. 8. Pre-main sequence evolutionary tracks calculated by Burrows et al. (1997) -left panel- and Chabrier et al. (2002) -right panel-, indicated with continuous lines, and superimposed on the HR diagram. The thick continuous line corresponds to $0.08 M_{\odot}\left(\sim 80 M_{J}\right)$, the $\mathrm{H}$ burning limit. The dashed lines show the isochrones calculated also by the same authors. The positions of four of the observed stars (see Table 4) are indicated with starred symbols.

$\Delta T \sim 150 \mathrm{~K})$ uncertainty. [SGR2003] 1 is a sub-stellar mass object regardless of the spectral type (or temperature) uncertainty. [KG2001] 102, [GK2001] 30, and [GK2001] 53 have masses close to the border line between the stellar and substellar regimes. An uncertainty of \pm 1 sub-type prevent us from establishing the (stellar or sub-stellar) nature of the objects (see Fig. 7). The rest of the sources have masses between 0.22 and $0.17 M_{\odot}$.

Luminosity determinations have a "typical" uncertainty of about a factor of 2 (or $\sim 0.03$ in log scale). However the effect on our mass determinations is less significant than the uncertainty in spectral type or effective temperature as the evolutionary tracks are almost vertical.

The masses for the objects for which near-infrared spectra are available agree within a factor of $\sim 2$ with those derived from the spectral classification based on near-infrared spectra, with the differences in the inferred masses reflecting the differences in the assigned spectral types.

Our sample sources span a range of ages between $\sim 10^{5} \mathrm{yr}$ and few $\times 10^{7} \mathrm{yr}$, in agreement with higher mass members of the cloud (Lawson et al. 1996). In particular, only a small fraction of our objects (two out of nine) have ages $\geq 10^{7}$ years, supporting the conclusions of Comerón et al. (2000) and Gómez \& Mardones (2003) on the star formation rate in the cloud.
For the four objects in Table 3 with masses close or below the H-burning limit we compare our mass and age determinations, based on the D'Antona \& Mazzitelli (1997) models, with two additional sets of pre-main sequence evolutionary tracks and isochrones. We select the models of Burrows et al. (1997) ${ }^{5}$ and Chabrier et al. (2002) ${ }^{6}$ as both include detailed calculations below the $0.08 M_{\odot}$ limit. Figure 8 shows these tracks and isochrones superimposed on the HR diagram. In Table 4 we compare masses and ages derived using these models. [SGR2003] 1, [GK2001] 30, and [GK2001] 53 have sub-stellar masses, independently of the adopted calculations, while [KG2001] 102 may be stellar or sub-stellar depending on the tracks used.

\section{Summary and conclusions}

We present optical spectra of eight candidate very low mass members and a previously known $T$ Tauri star of the Chamaeleon I dark cloud. All but two of these objects have

\footnotetext{
5 Available at

http://zenith.as. arizona. edu/ burrows/dat-html/data/

6 Available at

ftp: //ftp.ens-lyon.fr/pub/users/CRAL/ibaraffe/
} 
Table 4. Comparison of masses and ages corresponding to different models.

\begin{tabular}{l|lr|lr|ll}
\hline \hline Name & $\begin{array}{l}M / M_{\odot} \\
\text { B97 }\end{array}$ & Age [yrs] & $\begin{array}{l}M / M_{\odot} \\
{\mathrm{C} 02^{\mathrm{b}}}^{\mathrm{a}}\end{array}$ & Age [yrs] & $\begin{array}{l}M / M_{\odot} \\
\text { DM97 }\end{array}$ & Age [yrs] \\
\hline [KG2001] 102 & 0.027 & $<10^{5}$ & & & 0.09 & $4 \times 10^{5}$ \\
[SGR2003] 1 & 0.016 & $5 \times 10^{6}$ & 0.025 & $7 \times 10^{6}$ & 0.019 & $2 \times 10^{6}$ \\
[GK2001] 30 & 0.045 & $7 \times 10^{5}$ & 0.04 & $1 \times 10^{6}$ & 0.075 & $4 \times 10^{6}$ \\
[GK2001] 53 & 0.035 & $4 \times 10^{6}$ & 0.04 & $5 \times 10^{6}$ & 0.06 & $6 \times 10^{6}$ \\
\hline
\end{tabular}

${ }^{a}$ Burrows et al. (1997).

${ }^{\mathrm{b}}$ Chabrier et al. (2002).

${ }^{\text {c }}$ D'Antona \& Mazzitelli (1997).

$\mathrm{H} \alpha$ in emission with equivalent widths similar to other known very low mass $\mathrm{T}$ Tauri stars and previously identified young BDs (Lawson et al. 1996; Briceño et al. 1998; Neuhäuser \& Comerón 1998; Martín et al. 2001), indicating their young age. The Na I doublet (8183-8195 $\AA$ ) is weaker than in stellar objects of the same spectral type for [KG2001] 102, [GK2001] 30, and [GK2001] 31, supporting the very low mass nature of these objects (Martín et al. 1996). In the case of [GK2001] 53 the Na I doublet strength is comparable to two known BDs in the Pleiades. Higher resolution spectra of these objects would allow the detection of the $\mathrm{Li}$ I $\lambda 6707.8$ and thus an independent confirmation of their youth.

The lack of significant $\mathrm{H} \alpha$ emission in ISO-ChaI 237 and [GK2001] 8 casts doubts on the nature of these objects. The $\mathrm{Na}$ I doublet strength for [GK2001] 8 suggests that this object is probably a field dwarf. Higher resolution spectra are required to properly classify both ISO-ChaI 237 and [GK2001] 8.

In summary, out of eight candidates, two turned out not to be cloud members, the nature of other object ([GK2001] 53 ) would require additional confirmation by higher resolution spectroscopy, while $\mathrm{H} \alpha$, together with the $\mathrm{Na}$ I doublet strength in some cases, support the pre-main sequence nature and low mass classification of the remaining five sources.

We used the strength of the TiO or VO molecular bands as well as the PC3 index from Martín et al. (1999) and the grid of spectra of Kirkpatrick et al. (1991), to derive spectral types and adopted D'Antona \& Mazzitelli (1997) evolutionary tracks and isochrones to estimate masses and ages. We detect one new BD and three transition stellar/sub-stellar objects of the cloud. Burrows et al. (1997) and Chabrier et al. (2002) models confirm these results. The rest of the observed objects have masses between 0.22 and $0.17 M_{\odot}$. [SGR2003] 1 , with a derived mass of $0.019 M_{\odot}\left(\sim 19 M_{J}\right)$, lies very close to the deuterium burning limit $\left(\sim 13 M_{J}\right)$. This is the least massive object identified in the cloud so far. These sources span a range of ages between $\sim 10^{5} \mathrm{yr}$ and few $\times 10^{7} \mathrm{yr}$, in agreement with higher mass members of the cloud (Lawson et al. 1996).

The Chamaeleon I dark cloud has been searched at different wavelengths to detect sub-stellar mass objects during the last few years (Cambrésy et al. 1998; Oasa et al. 1999; Persi et al. 2000; Gómez \& Kenyon 2001). Comerón et al. (2000) reported the detection of four bona-fide BDs in the Chamaeleon I dark cloud in addition to eight candidate transition stellar/sub stellar objects (see also, Neuhäuser \& Comerón 1998; Neuhäuser et al. 2002). Gómez \& Persi (2002) and Gómez \& Mardones (2003) identified 30 very low mass objects, including several transition objects. These investigations have significantly increased the low mass population of the cloud (see Gómez \& Mardones 2003). However, these authors attempted a determination of the IMF of the cloud and noticed an apparent scarcity of very low mass objects in the whole Chamaeleon I cloud based on the behavior of the IMF in the central $300 \mathrm{arcmin}^{2}$ region, determined by Comerón et al. (2000). This small portion of the cloud has been intensively surveyed at different wavelengths combining both photometric and spectroscopic information. Gómez \& Mardones (2003) estimated that about 100 objects remain to be identified in the whole cloud.

The results presented in this paper contribute to the census of the pre-main sequence population of the cloud. In particular, we identified one sub-stellar object, increasing the number of bona-fide BDs in the cloud from 4 to 5. The newly discovered BD as well as the three transition objects analyzed in this contribution will eventually help to determine the behavior of the IMF for the complete cloud in the sub-stellar regimen and a comparison with other nearby star-forming clouds.

The newly detected objects increase the overall number of young BDs and transition objects known today. These objects will eventually allow us to carry out statistical studies of the physical properties of young BDs. To better understand similarities and differences among stars, brown dwarfs and planets, confrontations of the characteristics of the three groups are needed.

Acknowledgements. We are grateful to the ESO staff for assistance with the observations, specially to Martino Romaniello for help during the VLT Phase II preparation. We are grateful to Dr. Eduardo L. Martín, the referee, for helpful criticism that improved the content and presentation of this paper. This research has made use of the SIMBAD database, operated at CDS, Strasbourg, France. The CCD and data acquisition system at CASLEO has been partly financed by R. M. Rich trough US NSF grant AST-90-15827.

\section{References}

Briceño, C., Hartmann, L., Stauffer, J., \& Martín, E. L. 1998, AJ, 115, 2074 
Briceño, C., Luhman, K. L., Hartmann, L., Stauffer, J., \& Kirkpatrick, J. D. 2002, ApJ, 580, 317

Burrows, A., Marley, M., Hubbard, W. B., et al. 1997, ApJ, 491, 856

Cambrésy, L., Copet, E., Epchtein, N., et al. 1998, A\&A, 338, 977

Chabrier, G., Baraffe, I., Allard, F., \& Hauschildt, P. 2002, ApJ, 542, 464

Comerón, F., Neuhäuser, R., \& Kaas, A. A. 2000, A\&A 359, 269

Elmegreen, B. G. 1999, ApJ, 522, 915

D’Antona, F., \& Mazzitelli, I. 1997, Mem. Soc. Astron. It., 68, 807

Gómez, M., \& Kenyon, S. J. 2001, AJ, 121, 974

Gómez, M., \& Persi, P. 2002, A\&A, 389, 494

Gómez, M., \& Mardones, D. 2003, AJ, 125, 2134.

Hartigan, P., Kenyon, S. J., \& Hartmann, L. 1991, ApJ, 82, 617

Henry, T. J., Kirkpatrick, J. D., \& Simons, D. A. 1994, AJ, 108, 1437

Kenyon, S. J., Brown, D. I., Tout, C. A., \& Berlind, P. 1998, AJ, 115, 2491

Kenyon, S. J., \& Gómez, M. 2001, AJ, 121, 2673

Kirkpatrick, J. D., Henry, T. J., \& McCarthy, D. W. 1991, ApJS, 77, 417

Kirkpatrick, J. D., Henry, T. J., \& Simons, D. A. 1995, AJ, 109, 797

Lawson, W. A., Feigelson, E. D., \& Huenemoerder, D. P. 1996, MNRAS, 280, 1071

Magazzù, A., Martín, E. L., \& Rebolo, R. 1993, ApJ, 404, L17

Martín, E. L., Rebolo, R., \& Zapatero-Osorio, M. R. 1996, ApJ, 469, 706
Martín, E. L., Delfosse, X., Basri, G., et al. 1999, ApJ, 118, 2466

Martín, E. L., Dougados, C., Magnier, E., et al. 2001, ApJ, 561, L195

Muench, A. A., Lada, A. E., Lada, C. J., \& Alves, J. 2002, ApJ, 575 354

Natta, A., \& Testi, L. 2001, A\&A, 376, L22

Neuhäuser, R., \& Comerón, F. 1999, A\&A, 350, 612

Neuhäuser, R., Brandner, W., Alves, J., Joergens, V., \& Comerón, F. 2002, A\&A, 384, 999

Oasa, Y., Tamura, M., \& Lawson, W. A. 1999, ApJ, 526, 336

O'Connell, R. W. 1973, AJ, 78, 1074

Oppenheimer, B. R., Kulkarni, S. R., \& Stauffer, J. R. 2000, in Protostars and Planets IV (Tucson: The University of Arizona Press), ed. V. Mannings, A. P. Boss, \& S. S. Russell, 1313

Persi, P., Marenzi, A. R., Olofsson, G., et al. 2000, A\&A, 357, 219

Pickett, B. K., Durisen, R. H., Cassen, P., \& Mejia, A. C. 2000, ApJ, 540, L95

Reipurth, B., \& Clarke, C. 2001, AJ, 122, 432

Rieke, G. H., \& Lebofsky, M. J. 1985, AJ, 288, 618

Schwartz, R. D. 1977, ApJS, 35, 161

Whittet, D. C. B., Prusti, T., Franco, G. A. P., et al. 1997, A\&A, 327, 1194

Wilking, B. A., Greene, T. P. \& Meyer, M. R. 1999, AJ, 117, 469 